\title{
Generic Control Interface for Networked Haptic Virtual Environments
}

\author{
Priscilla Ramsamy, Adrian Haffegee, and Vassil Alexandrov \\ Advanced Computing and Emerging Technologies Centre, \\ The School of Systems Engineering, University of Reading, \\ Reading, RG6 6AY, United Kingdom \\ p.ramsamy@reading.ac.uk
}

\begin{abstract}
As Virtual Reality pushes the boundaries of the human computer interface new ways of interaction are emerging. One such technology is the integration of haptic interfaces (force-feedback devices) into virtual environments. This modality offers an improved sense of immersion to that achieved when relying only on audio and visual modalities. The paper introduces some of the technical obstacles such as latency and network traffic that need to be overcome for maintaining a high degree of immersion during haptic tasks. The paper describes the advantages of integrating haptic feedback into systems, and presents some of the technical issues inherent in a networked haptic virtual environment. A generic control interface has been developed to seamlessly mesh with existing networked VR development libraries.
\end{abstract}

Keywords: Virtual Reality, Force Feedback.

\section{Introduction}

"The real power of VR technology is that it allows people to expand their perception of the real-world in ways that were previously impossible" [1. Changes to virtual objects and their interrelationships can be effected with ease, which is often not feasible with real objects. Through VR market researchers are now able to generate precisely the same stimulus conditions for all participants and are able to modify certain environment variables in real-time thus allowing them to have more control over the experimental settings. Our study is centred on exploring shoppers' habits for market research with regards to how they move about in their search for desired items within the shopping centre. Based on these results market researches can provide the relevent foundation for developing effective marketing strategy and advertising campaigns to boost sales. A random sample of people categorised by age was selected and were interviewed about their experience regarding the efficiency, ease of use and degree of realism to the physical world that the application had. Analysis of the collected data showed that respondents not only experienced difficulty to navigate in the 3D environment using the wand but also in its use to actually get hold of certain objects by pressing buttons. It was then decided to develop a more natural and 
intuitive mode of interaction and navigation to better suit the users in such an environment.

Input devices such as computer keyboards, mice and joysticks can convey the user's commands to the computer but are unable to provide a natural sense of touch and feel to the user. It is crucial to understand the nature of touch interaction, since we react to our surroundings based on what and how we perceive things [2]. The user's sensorial experience in a virtual environment is a fusion of information received through his perception channels. The results obtained from previous studies have made it possible to quantify the effectiveness of using haptics to provide a greater sense of presence and immersion. Based on these findings 3] 4 [5] 6 there is great potential to improve VR applications. The additional information from haptic feedback makes certain tasks such as manipulation much easier compared to that of the traditional computer interface [7. In an effort to bring in more realistic and intuitive experiences in the virtual environment created, a haptic trolley and an instrumented glove were incorporated with the VE. The study consists of augmenting an immersive graphic display (CAVE like system 8]) with a haptic interface.

The integrated system is multi-frequency since the haptic loop and the display/rendering loop require different update rates. The haptic loop should be maintained with an update rate of $1 \mathrm{KHz}$ to avoid force artifacts and because humans can detect discrete events at less than this rate. The display loop should be updated at $30 \mathrm{~Hz}$ (minimum) to produce stable images and since the human visual system has a flicker frequency around $30-60 \mathrm{~Hz}$. An effective integrated system of haptics and graphics should ensure the effectiveness of each modality. The users experience and sense of immersion would be enhanced and would complement the visual display only if both the haptic and the visual feedback are consistent with each other. Evidence show that latency is a limiting factor in shared haptic environments 9. Latency creates a lag from when collision is detected and when a force is applied to the display device. The latency experienced for local systems is very small(instantaneous) however, for systems that are distributed across a network the lag experienced could generate instability during haptic rendering. A generic control interface has been developed at Reading University which forwards the relevant device information over the network to both the haptic and rendering loop. The latency felt is minimal as we are integrating the haptic display on a local system.

In section 2 of this paper, we give an overview of the software architecture implemented as well as the networking protocol used and how its implementation has been utilised in real-time. Section 3, presents the current feedback device operations developed. Finally the future work is outlined in section 4 .

\section{Software Architecture}

Haptic simulations involve a human operator, a force reflecting device and a virtual environment. Haptic is a term derived from the Greek "haptesthai" which means "to come in contact with" by providing the sense of touch with both tactile 
(cutaneos) and kinaesthetic (proprioception) feedback [10]. Tactile, or touch feedback describes the sensations felt by the skin. Tactile feedback permits users to feel things such as the texture of surfaces, temperature and vibration. kinaesthetic force feedback on the other hand enables us to recognise a force that is applied to our body by the help of sensoric cells located at the end of the tendons or between the muscle strands. The human operator kinaesthetically explores the virtual environment by interacting and grasping an active mechanical device. The device provides a new sensory display modality and presents the necessary information by exerting controlled forces on the human operator. Figure 3. shows the mobile platform developed. The device is equipped with an actuator that produces the relevant forces and a sensor from which the velocity can be calculated. The motion and position of the device is coupled to a virtual object in the virtual environment. The virtual object should experience the same forces and movement as experienced by the physical device.

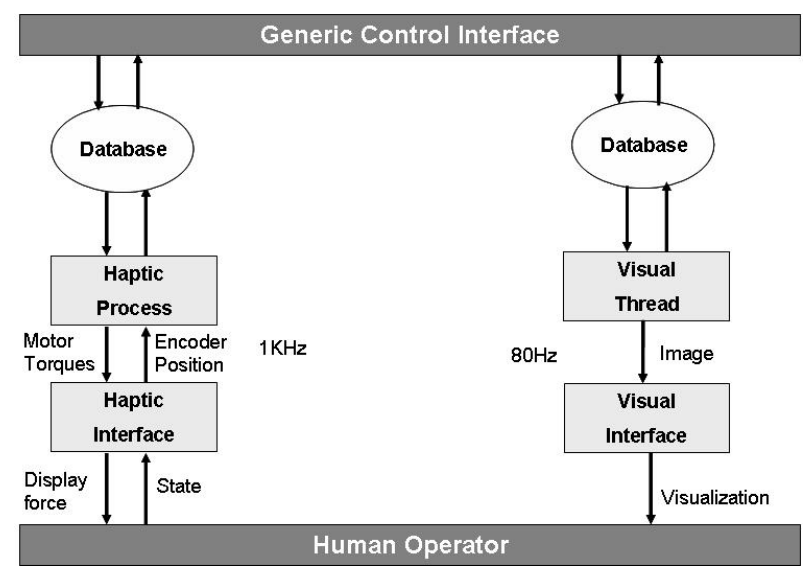

Fig. 1. Software architecture for multi-processing

Several issues can contribute to limiting haptic device capability to render and provide the relevant forces. To provide the desire results the system should update in real-time however to have higher accuracy would require that we sacrifice the update speed. Therefore due consideration should be given between the trade-offs of being highly efficient or highly accurate.

The generic interface in Figure 1, currently forwards the relevant information that is velocity, force and position over the network. These values are then used by both the visual-rendering and haptic-rendering loop to produce the necessary visual and haptic feedback. In our implementation the haptic and the graphic loops have their own databases, which are not shared. This architecture enables us to run the haptic process and visual process on either different computer systems or on the same system. For this multi-processing structure the critical task would be to update both the graphic and the haptic database. The visual 
rendering subsystem displays the necessary scenes onto the display system and this is usually run at rates of up to $80 \mathrm{~Hz}$. For this work the VieGen framework as described in [1] was used for control and rendering of the scene.

\subsection{Transport Protocol Used}

Since the transmission speed would have a direct bearing on the update rate of the simulation engine the solution chosen was a trade-off to provide maximum flexibility and high throughput at the expense of the implementation being unreliable. Currently the User Datagram protocol (UDP) transport protocol is used to route data packets over the network. However based on the fact that the network currently being used is a close coupled controlled network, reliability due to lost packets is not be an issue. Error recovery for lost packets has been inbuilt in the system. The interface is written in $\mathrm{C}$ programming language and uses the stream socket or connected datagram socket for sending and receiving data across the network.

\subsection{The Client/Server Architecture}

The proposed design is a client/server architecture. The real-time implementation is done over a LAN and consists of 3 components namely the client, the server and the generic control interface. The server component: is responsible for receiving packets from the force reflecting/haptic device through the $\mathrm{xPC}$ TargetBox. The server is then responsible for forwarding the relevant information over the network. A generic control interface deals with this information by writing and storing it into shared memory on each system. The following flowchart Figure 2, depicts the current workings of the server application. This application unpacks the packets received, which consists of data for both the instrumented glove and the mobile platform. Once the data is unpacked the information is stored in the relevant structure in shared memory. Calls are made to the underlying methods in the generic control interface to write to shared memory. Error checks have been implemented to verify whether memory has been initialised. If not, a memory segment is initialised and allocated to accommodate the information for both the instrumented glove and the mobile platform data structures. Since the packets sent over the network are updated at a high frequency writing to shared memory should be synchronised to be able to update the information at the same rate. The client component: is responsible for retrieving the relevant information from memory and using it to render scenes in the Virtual environment. The update/refresh rate can be set according to the application requirement. The server component receives data from the haptic interface via the network whilst the client interface sends the force values back to the haptic interface. Both Client/Server components run on the same host. As mentioned previously the motion and position of the haptic trolley is coupled to a virtual trolley object in the virtual environment. The virtual object should experience the same forces and movement as experienced by the physical device. Hence to provide a realistic experience to end-users the scene rendered 

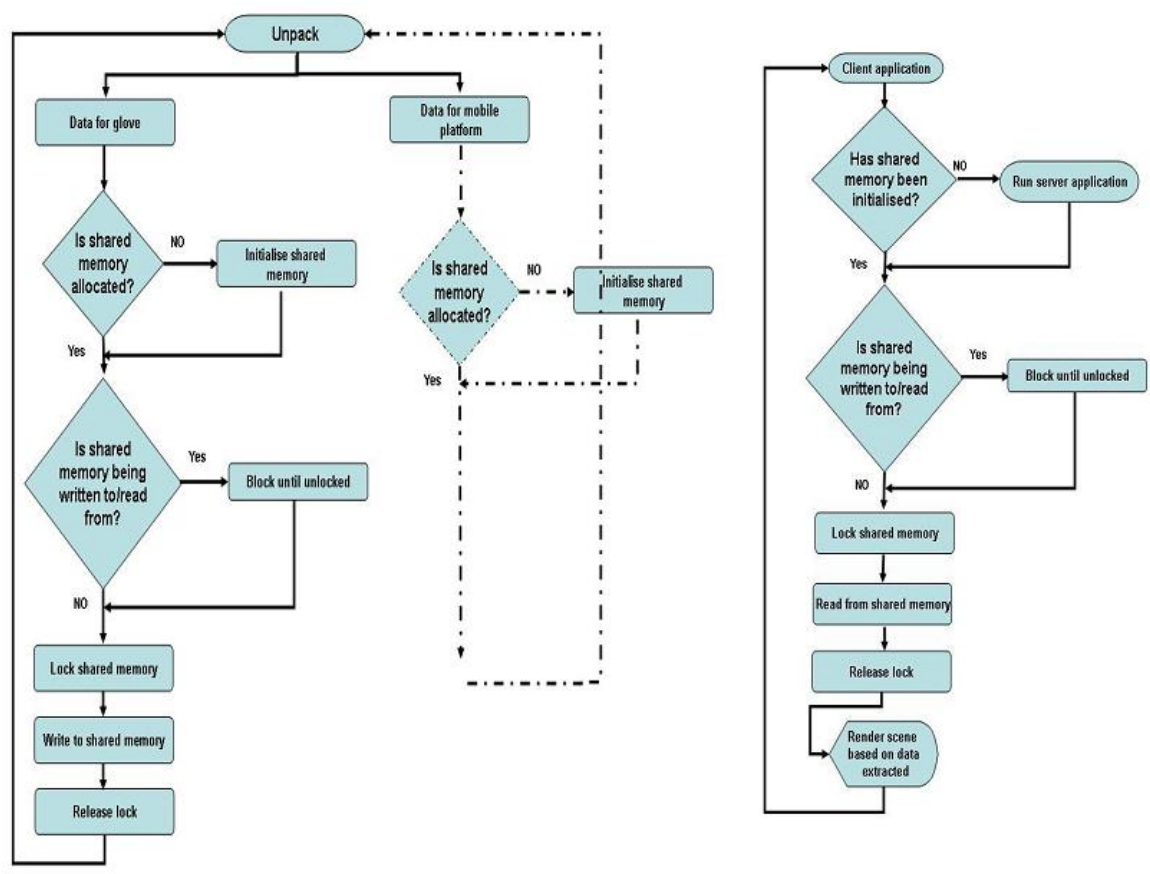

Fig. 2. Server and Client Application Flowchart

should portray the changes in real-time and this is achieved by enabling a high refresh/update rate. The interface was tested and produced successful results as to providing a realistic enhanced experience to the end-user. Since the current network implementation was based on the User Datagram protocol less overhead and network lag was experienced. We are currently experiencing a round trip time of $1 \mathrm{~ms}$ for every packet being sent whilst the grounded trolley is being used as a navigational tool. The flowchart in Figure 2] depicts the current workings of the client application.

\subsection{Application Development}

The shopping application was developed to allow users in the ReaCTor to shop in the virtual environment by interacting with $3 \mathrm{D}$ models of different products and brands. A more intuitive mode of navigation was provided by integrating the trolley during user trials. The application implemented made use of the VieGen framework as described in [10]. VieGen provides several libraries of tools and utilities that are useful in the development of Virtual Interactive Environments. The elements making up the framework consists of the interface to the display hardware, a networking subsystem, scene management, environment simulation and accompanying utilities, all of which can be used or left out as depending on 
the requirements. The CAVE Scene Manager component (CSM) offers a generic abstraction layer to the underlying VR hardware and this allows the same application to be compiled for a variety different systems. At run-time a configuration file is then used to stipulate the required system set-up and mode of operation and by using this approach, it was possible to use the VieGen framework to run on different display systems. Ranging from mono desktop systems through Powerwalls and through to immersive CAVE like systems. With such an application, it not only implies application interoperability throughout the entire range of hardware systems, but it also lends itself to application development and testing without the excessive tying up of the essential immersive system resources.

The networking subsystem consists of two layers. While the low level layer provides a consistent, low latency infrastructure for buffering messages to and from multiple remote locations, the higher level layer [9] builds on top of this to provide a hierarchical topology for Distributed Virtual Environments (DVEs). This topology assumes that the environment is split into domains and controls the individual users in these domains. One of the users is dynamically and transparently allocated (designated) as the Domain Server, and is consequently used by the topology to handle connections between the remaining clients. Later these connections are used to relay information such as position, action events or user-defined messages. The modules for simulation are run concurrently with the networking subsystem. This operation forms the basis for establishing an environment as a domain, and allows its population by a number of computer generated users. It is then possible for these pseudo-users to perform actions within the environment to collect statistical data, perform tests and statistical analysis.

\section{Device Operations}

In our implementation we have a instrumented tactile glove and a grounded haptic mobile platform both are currently being developed at Reading University. The tactile glove can replace or be used in conjunction with the traditional wand to provide a more realistic and intuitive way of manipulating virtual objects. The generic control interface developed permits function calls to the device state whereby the relevant information can be retrieved. The existing functions can retrieve the hand orientation and finger angles. The appropriate feedback can be rendered based on these values retrieved and the chosen collision detection algorithm. Once collision is detected with a manipulatable object the person would be able to interact with and manipulate the object. The virtual object will be attached to the glove only while the grasp aperture (the distance between thumb and fingers) is less than a critical distance. The critical distance can be fixed or vary depending on the size of the object being manipulated. The following figure 3 shows the glove in question being used in the immersive shopping environment to provide a more intuitive way to manipulate objects in the environment. The haptic mobile platform is currently being used to replace 


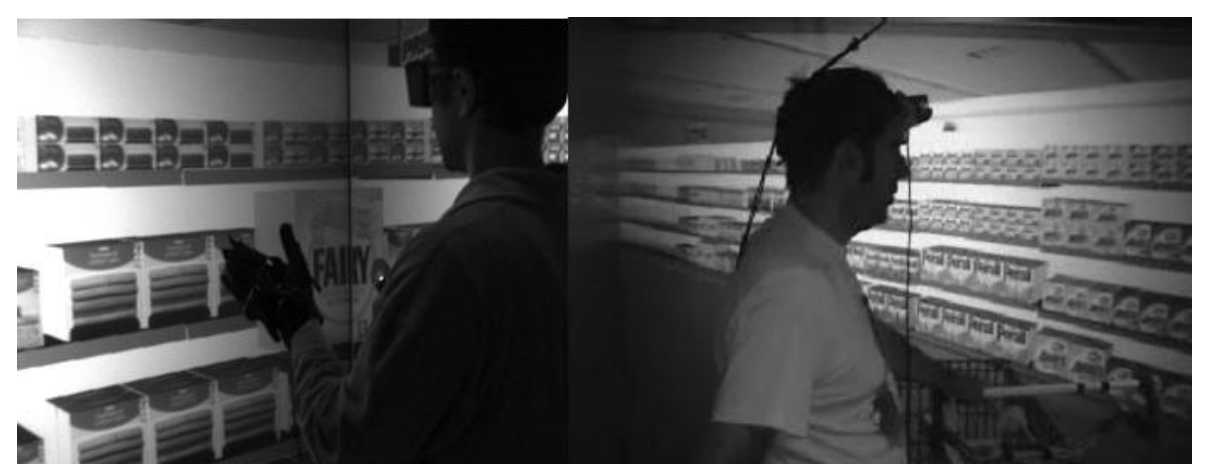

Fig. 3. Glove and trolley in use

the traditional joystick and permits the user to navigate within the virtual environment. It could mimic several devices ranging from a bicycle to a trolley or hang glider. Information on the device sate, the force applied, the displacement forward or backwards and the angle of rotation can be retrieved. Figure 3 shows the trolley being used as a navigational tool in the shopping environment.

\section{Conclusion and Future Work}

Our generic control interface has been successfully developed and permits both the tactile and force feedback devices to integrate with our immersive system, and is being used in ongoing application development. The current work in progress involves making the generic interface a platform independent portable interface. The generic control interface provides a flexible method of developing libraries that acts as an interface between different input devices and existing CVEs.

Based on the requirements for stability and fidelity a very high update rate has been used in our current implementation. However to minimise transmission requirements updates need only be made when there is a change in state. Our future work will concentrate in implementing such an architecture to enable an improved local interaction and to find the effects on the degree of immersion and haptic feedback of the system. The magnitude of inconsistencies between states of the local copies of the VE will be evaluated to verify the network delay and the amount of packet loss. Refinement of current haptic device operations and incorporating an increased number of vibrotactile stimulators onto each finger of the glove would also be considered. This would enhance and provide more functionality to the instrumented glove would enable a realistic mode of interaction in VEs.

The current implementation considers the integration of haptic information to augment visual displays on a local system. The latency experienced is minimal in such a situation. However the work will be extended to provide a haptically 
mediated collaborative virtual environment. This would be challenging considering that the users would be graphically distributed and various problems such as network delay and latency would be introduced.

\section{References}

1. J.L. Mohler, "Desktop Virtual Reality for the Enhancement of Visualization Skills". Journal of Educational Multimedia and Hypermedia,(2000) 151-165.

2. M.A. Srinivasan and C. Basdogan, "Haptics in Virtual Environments: Taxonomy, Research Status, and Challenges," Computers and Graphics, Special Issue on Haptic Displays in Virtual Environments, Vol. 21, No. 4, (1997)

3. Burdea, G.C. Haptics issues in virtual environments. In Computer Graphics International,Proceedings. (2000)

4. F. P. Brooks, M. Ouh-Young, J. J. Batter, and P. J. Kilpatrick. Project GROPE - Haptic Displays for Scientific Visualization. In Proc. ACM SIGGRAPH, Dallas, TX, Aug (1990) 177-185

5. Michael Dinsmore, Noshir Langrana, Grigore Burdea, Jumoke Ladeji. "Virtual Reality Training Simulation for Palpation of Subsurface Tumors," vrais, Virtual Reality Annual International Symposium, (1997) 54

6. Burdea, G., G. Patounakis, V. Popescu, \& R. E. Weiss. Virtual Reality Training for the Diagnosis of Prostate Cancer. In IEEE International Symposium on Virtual Reality and Applications, Atlanta, Georgia, March (1998) 190-197

7. Andrew G. Fischer, Judy M. Vance, "Implementing Haptic Feedback in a Projection Screen Virtual Environment," Seventh PHANToM Users Group Workshop, October (2002) 26-29

8. Cruz-Neira, C.,Sandin, S.J.,DeFanti, T.A., Kenyon, R.V., and Hart, J.C. The CAVE: Audio visual experience automatic virtual environment. Communications of the ACM 35 (1992) 64-72

9. Ottensmeyer MP, Hu J, Thompson JM, Ren J, and Sheridan TB Investigations into performance of minimally invasive telesurgery with feedback time delays. Presence 9 (4), (Aug 2000) 369-382.

10. M. Mokhtari, F. Bernier, F. Lemieux, H. Martel, J.M. Schwartz, D. Laurendeau and A. Branzan-Albu. Virtual Environment and Sensori-Motor Activities: Haptic, Audition and Olfaction. WSCG POSTERS proceedings Plzen, Czech Republic., Vol.12, No.1-3, February, (2004) 2-6

11. Haffegee, A., Jamieson, R., Anthes, C., Alexandrov, V. " Tools for Collaborative VR Application Development." In: International Conference on Computational Science, Springer Verlag (2005) 350358 